\title{
Emergence of Zika virus in India: Implications for pregnant women and obstetrician
}

\author{
Moushmi B. Tadas Parpillewar \\ Correspondence: Dr Moushmi B. Tadas Parpillewar. Associate Professor, Department of \\ Obstetrics and Gynaecology, Government Medical College, Nagpur, Maharashtra, India. Email - \\ drmoushmi@yahoo.co.in
}

Distributed under Creative Commons Attribution-Share Alike 4.0 International.

\begin{abstract}
With the emergence of first case of Zika virus infection in India, it is very important to know the consequences of this infection on the pregnant women and her foetus. At the same time the obstetrician and her treating physician must have knowledge of this infection, its etiopathogenesis and treatment. Although this infection causes mild symptoms in humans, it causes transmission from mother to fetus leading to congenital infection to the fetus leading to microcephaly. It is transmitted to humans via bite of infected aedes mosquito, vector which is abundant in India and the vulnerable population with very little or no immunity for this infection. Zika virus is closely related to yellow fever, dengue and Japanese encephalitis viruses but causes mild variety of symptoms which are never fatal. But reports and evidences from the epidemic of microcephaly in infants born to mothers suffering from Zika virus infection in Brazil led to the conclusion that this microcephaly is caused by congenital Zika virus infection. There is no vaccine for this infection, treatment is symptomatic. Diagnosis is by serological testing.
\end{abstract}

Keywords: Zika virus, microcephaly, aedes mosquito, amniocentesis, RT-PCR.

“On May 15, 2017, the Ministry of Health and Family Welfare, Government of India, reported three laboratoryconfirmed cases of Zika virus disease in Bapunagar area, Ahmedabad district, Gujarat," says the WHO report dated May 26, 2017. ${ }^{1}$ This report is important as it describes the first case of Zika virus (ZIKAV) infection in India, one case of the three being pregnant, and provides evidence on the circulation of the virus in India. These findings suggest low level transmission of ZIKAV and new cases may occur in the future. With the emergence of Zika infection in India, although it causes mild symptoms in humans, it results in transmission from mother to fetus leading to congenital infection to the fetus leading to microcephaly. Now as the vector for transmission of ZIKAV i.e. the Aedes mosquito are present in abundance in India, the vulnerable pregnant population with zero immunity, is at risk for getting infected and delivering a malformed baby. As it lead to an epidemic of Zika related microcephaly in Brazil, sensitization regarding this infection is the need of the hour in India to prevent the microcephaly. So this review tries to give a brief overview of the ZIKAV, its modes of transmission, symptoms, diagnosis, treatment and its prevention.

What is Zika virus?

It is a single stranded RNA virus of genus Flavivirus, family Flaviviridae. It is closely related to dengue, yellow

Received: $29^{\text {th }}$ October 2017. Accepted: $10^{\text {th }}$ December 2017.

Tadas Parpillewar MB. Emergence of Zika virus in India: Implications for pregnant women and obstetrician. The New Indian Journal of OBGYN. 2018; 4(2): 100 - 5 
fever, Japanese encephalitis and West Nile viruses. It is transmitted to humans primarily by Aedes species of mosquitoes. It was first identified in monkeys in Zika forest in Uganda in the year 1947. ${ }^{2}$ Thereafter, sporadic human cases have been reported in parts of Africa, the Americas, South East Asia and Western Pacific. ${ }^{3}$ In 2007, first outbreak of Zika was documented from the Pacific region. Subsequently, outbreaks have been reported from Africa, America, Asia, French Polynesia, Western Pacific and Brazil ${ }^{4}$. Since 2014, indigenous circulation of ZIKAV has been detected in the Americas. Alarm bells started ringing in October, 2015 in Brazil, when association of ZIKAV was suspected with babies born with microcephaly, an abnormally small head often with consequent brain damage. ${ }^{1,5}$

\section{Modes of Transmission}

Commonly it is transmitted by female Aedes mosquito bite from infected to uninfected humans and primates. Various species of aedes mosquito may have the potential to transmit Zika, but the most commonly associated with ZIKV is Aedes aegypti ${ }^{6}$. This type of mosquito usually bites during the daytime and breeds in standing water. It can also be transmitted from mother to fetus transplacentally. Other proposed modes of transmission are sexual, ${ }^{7,8}$ via blood transfusion, theoretical organ or tissue transplantation, and breast milk. ${ }^{9}$

\section{Clinical disease course, symptoms and outcomes}

After an infected mosquito bites a human, the first symptoms of ZIKV can develop in 3 to 12 days but it can be shorter or longer in some people. The infection rate after mosquito bite is 73\% (95\% CI 68-77). Symptomatic attack rate among the infected person is $18 \%(95 \% \mathrm{Cl} 10$ 27). All age groups can be affected. The disease course is not severe, does not require hospitalization and deaths have not been reported ${ }^{10,11}$. Most common symptoms are a maculopapular rash, fever, arthralgia, conjunctivitis, myalgia, and headache. These remain for several days to a week. Fatalities are rare, typically only with comorbidities. However, an increase in cases of fetal microcephaly, ${ }^{1}$ Guillain-Barre syndrome and other neurological and autoimmune syndromes has been reported in areas where ZIKV outbreaks have occurred. ${ }^{12}$ Although these associations cannot yet be said to be causal, emerging evidence suggests that they may be. In November 2015, four perinatal deaths from ZIKV infection associated with microcephaly were reported in Brazil. $^{13}$

\section{Diagnostic Testing for Zika virus}

The mainstay of detection of ZIKAV in maternal serum is Reverse Transcriptase Polymerase Chain Reaction (RT- PCR) assay to be done to detect viral RNA in serum and urine collected $\leq 7$ days after illness onset. Serological assays to detect either IgM or IgG in serum collected $\geq 4$ days after illness onset can also be done. But antibody testing is less reliable due to potential crossreaction with antibodies against other similar viruses (e.g. dengue or yellow fever) making it difficult to differentiate ZIKV infection using antibody testing alone. ${ }^{14}$ Plaque reduction neutralization test (PRNT) is done to detect $\mathrm{a} \geq$ 4 - fold rise in ZIKAV specific neutralizing antibodies. ZIKV RT-PCR can also be performed on amniotic fluid ${ }^{15}$ although it is currently not known how sensitive or specific this test is for congenital infection, or the likelihood of an infected fetus being affected, i.e. subsequently developing a fetal abnormality. In India NCDC, Delhi and National Institute of Virology (NIV), Pune, have the capacity to provide laboratory diagnosis of ZIKAV disease in acute febrile stage.

\section{Zika Virus and Pregnancy}

Limited information is available on this subject. But existing data shows that infection can occur in any trimester, ZIKAVcan cross the placental barrier, and it is likely that Zika infection in early pregnancy poses the greatest risk. There is no evidence of increased susceptibility to Zika infection during pregnancy. There is no evidence of more severe disease.

\section{Zika virus and congenital infection}

In October 2015 Brazil reported an association between ZIKAV infection and microcephaly. For this event causal link was not proven. Between March 2015 and March 2016, more than 5000 cases of microcephaly have been reported among newborns born to Brazilian mothers with ZIKAV infection, there was >20-fold increase in microcephaly compared with previous years ${ }^{16}$. ZIKAV was been detected in blood and tissues of affected foetuses and infants. The mothers of these cases presented with symptoms consistent with ZIKAV infection during pregnancy and they tested positive for the infection. ${ }^{15,17}$ Most of them had infection in first or second trimester. One study of 35 cases of microcephaly in Brazil found 
that $26(74 \%)$ of the women reported having had a rash, 21 in the first trimester, 5 in the second trimester and none in the third trimester. ${ }^{18}$ Based on this information and on experience from other congenital infections such as CMV, rubella and toxoplasmosis, it is likely that ZIKAV infection in early pregnancy poses the greatest risk. ${ }^{19}$ Congenital ZIKAV infection leads to fetal microcephaly, fetal loss (in the first trimester), fetal growth restriction, placental insufficiency, fetal death, ocular involvement macular atrophy and optic nerve abnormalities and hydrops fetalis. ${ }^{19}$

\section{Zika virus related microcephaly}

ZIKAV-related microcephaly has been defined by the WHO as fetal microcephaly with a molecular or epidemiologic link to ZIKAV, in the absence of other conditions known to cause microcephaly. ${ }^{20}$

A molecular or epidemiologic link to ZIKAV is defined as one of the following: ${ }^{20}$

- Mother had confirmed case of ZIKAV infection during pregnancy

- Mother had sexual contact during pregnancy with a person with confirmed ZIKAV infection

- Mother had typical clinical manifestations of ZIKAV infection (two or more of the following: fever, maculopapular rash, arthralgia, or conjunctivitis) and relevant epidemiologic exposure during pregnancy (residence in or travel to an area where mosquito-borne transmission of ZIKAV infection has been reported)

- Amniocentesis with detection of ZIKAV in amniotic fluid via PCR

- Postmortem detection of ZIKAV in fetal brain tissue via PCR

\section{Prevention}

There is no vaccine for prevention of this infection. Pregnant women should take measures to avoid mosquito bites for personal protection as well as take environmental control measures. Repellent products must be used while indoors or outdoors. Women must dress themselves in long-sleeved shirts and long pants to avoid mosquito bites. Aedes mosquitoes are active during the day so must stay indoors during that time. Mosquito nets must be used if sleeping during daytime. Recommended repellent products are preparations with $\mathrm{N}$-diethyl meta toluamide (DEET) which are up to $50 \%$ safe in pregnant and breastfeeding women. ${ }^{21,22}$ Others are picaridin, IR3535.Insect repellents should be applied regularly, particularly after swimming and in hot humid conditions when they may be removed by perspiration. When both sunscreen and insect repellents are required, the insect repellent should be applied over the sunscreen. Not recommended repellents are Citronella Oil based repellents, vitamin $\mathrm{B} 12$ complex, vitamin $\mathrm{B} 1$, and tea tree oil repellents. ${ }^{21}$ Environmental control measures include identification and elimination of potential mosquito breeding sites. Mosquito larvae breed in standing water; therefore, standing water must not be allowed to collect outdoors.

\section{Prenatal fetal evaluation}

Tools for fetal evaluation of ZIKAV infection are serial ultrasound examinations and amniocentesis.

\section{Serial ultrasound}

Serial ultrasound (every three to four weeks) is done in pregnant women with positive or inconclusive test results. ${ }^{23}$ Ultrasound findings consistent with ZIKAV microcephaly(suggested by head circumference more than two standard deviations below the mean) and intracranial calcifications (cerebellum, intraocular, brain). ${ }^{24}$ These findings may be detected as early as 18

to 20 weeks of gestation. If the laboratory tests are negative in a pregnancy below 20 weeks, screening ultrasound examinations are done at 20 and 28 weeks of gestation. After 20 weeks of gestation, screening ultrasound examinations are done about two and six weeks later. If ultrasound shows abnormality, amniocentesis is offered. ${ }^{25}$ In positive or inconclusive laboratory test results or symptomatic infection prior to 20 weeks of gestation, serial ultrasonography is done every 2 to 4 weeks starting at 18 weeks of gestation. After 20 weeks of gestation, serial ultrasonography should start at the time of ZIKAV infection diagnosis. ${ }^{25}$

\section{Amniocentesis}

Amniocentesis is offered when ultrasound findings of fetal microcephaly, intracranial calcifications, (regardless of maternal laboratory test results for ZIKAV infection) are present. It is also done if maternal laboratory testing for ZIKAV infection is positive or inconclusive and at least 6 to 8 weeks after maternal exposure. ${ }^{20,26}$ The optimal timing for performance of amniocentesis is 
uncertain. Sensitivity is higher if done $\geq 21$ weeks. Optimal timing is at 15-16 weeks in lab positive women. If negative then it may be repeated and other causes of microcephaly should be evaluated. Not recommended to do it before 14 weeks for fear of pregnancy loss.

\section{Post natal evaluation}

For a live birth with evidence of maternal or foetal ZIKAVinfection, tests recommended are: ${ }^{27}$

- Histopathologic examination of the placenta and umbilical cord;

- Testing of frozen placental tissue and cord tissue for Zika virus RNA;

- Testing of cord serum for Zika and dengue virus IgM and neutralizing antibodies.

If a pregnancy results in a foetal loss in a woman with history of travel to an area of ZIKAV transmission with symptoms consistent with ZIKAV disease during or within 2 weeks of travel or findings of foetal microcephaly, tests recommended are ${ }^{26}$

- Zika virus RT-PCR and immunohistochemical staining on foetal tissues, including umbilical cord and placenta.

\section{Infants with microcephaly}

For the purpose of evaluating an infant for possible congenital ZIKAV microcephaly is defined as occipitofrontal circumference greater than two standard deviations below the mean or less than the third percentile based on standard growth charts for sex, age, and gestational age at birth ${ }^{28,29}$ and not explained by other aetiologies or congenital disorders. If an infant's occipitofrontal circumference is equal to or greater than the third percentile but is notably disproportionate to the length of the infant, or if the infant has deficits related to the central nervous system, additional evaluation for ZIKAV infection may also be appropriate.

An infant with microcephaly with positive or inconclusive test results for ZIKAV infection should have additional assessment for possible long-term sequelae hearing loss, neurological screening. ${ }^{27}$

An infant with microcephaly or intracranial calcifications and negative results on all laboratory ZIKAV tests should be evaluated for alternative etiologies.

\section{Infants with no microcephaly}

Infants born to a mother potentially infected with ZIKAVduring pregnancy depends on the results from maternal testing. ${ }^{27}$

- Whose mother had negative laboratory test results should receive routine care.

- Mother had positive or inconclusive laboratory test results, infants should be tested for ZIKAV.

- If all of the infant's laboratory test results are negative, the infant should receive routine care.

- If any of the infant's laboratory test results are positive or inconclusive, the infant should undergo further clinical evaluation for long term sequelae, hearing loss, occipitofrontal diameter and developmental milestones. ${ }^{29}$

Intrapartum infection is suspected in an infant during the first two weeks of life whose mother had relevant epidemiologic exposure within two weeks of delivery and who has $\geq 2$ of the following manifestations: fever, rash, conjunctivitis, or arthralgia. Lab testing is done in both mother and newborn.

Transmission of ZIKAV through breastfeeding has not yet been described, further study is needed. Some have recommended that women continue to breastfeed. No developmental complications have been observed in otherwise healthy neonates, infants, or children with postnatal ZIKAV infection or exposure; further study is needed.

\section{Treatment}

There is no vaccine or any specific antiviral treatment. Treatment is mostly supportive rest, fluids, and use of analgesics and antipyretics. Fever is treated with acetaminophen. ${ }^{30}$ Aspirin and other nonsteroidal antiinflammatory drugs are avoided until dengue infection has been ruled out, to reduce the risk of hemorrhage. Some infected fetuses may be at risk for stillbirth. If antenatal testing is performed (eg. nonstress test, biophysical profile) and results are abnormal, early delivery may be indicated.

Pregnant women residing in areas with on-going Zika virus infection

Symptomatic pregnant residents should be tested for ZIKAV infection during the first week of symptoms. Asymptomatic pregnant residents may be offered screening with serologic testing at the initiation of prenatal care and mid-second trimester. ${ }^{31}$ These women 
must use personal protective measures to prevent mosquito bite.

\section{Women in areas of on-going Zika viral transmission} who desire to become pregnant

Such women must prevent mosquito bites. They must be provided with preconception care, which includes a discussion about the potential risk of ZIKAV infection in pregnancy, the signs and symptoms associated with ZIKAV disease, and when to seek care. ${ }^{31}$

Women planning pregnancy after travel from Zika infected area

Such women should avoid pregnancy immediately and for further 28 days, this allows for a maximum two week incubation period and possible two-week viraemia.

Women whose partner has been to an area with Zika transmission

The risk of sexual transmission of Zika is very low. ZIKAV are identified in semen of men who have had infection. Effective contraception is advised to for 28 days after his return home if he had no Zika symptoms, either whilst abroad or within 2 weeks of his leaving the affected country. Pregnancy should be avoided for 6 months following recovery if he did experience Zika symptoms during that period. ${ }^{31}$

Guidelines by Ministry of Health and Family Welfare and Directorate General of Health Services of India for international travellers to Zika infected areas:

- Non-essential travel to the affected countries to be deferred/ cancelled.

- Pregnant women or women who are trying to become pregnant should defer/ cancel their travel to the affected areas.

- All travellers to the affected countries/ areas should follow individual protective measures, to prevent mosquito bites.

- Persons with co-morbid conditions (diabetes, hypertension, chronic respiratory illness, Immune disorders etc.) should seek advice from the nearest health facility, prior to travel to an affected country.

- Travellers having febrile illness within two weeks of return from an affected country should report to the nearest health facility.

Pregnant women who have travelled to areas with ZIKAV transmission should mention about their travel during ante-natal visits in order to be assessed and monitored appropriately.

\section{Conflict of interest: None. Disclaimer: Nil.}

\section{References}

1.WHO. Zika virus infection - India. Disease Outbreak News. Geneva: WHO; 2017.

2.Centres for Disease Control and Prevention. Zika Virus. [Internet]. 1600 Clifton Road Atlanta; US Department of Health and Human Services. 2017. [Cited 2017 June 2]. Available from: http://www.cdc.gov/zika/geo/index.html.

3.Lanciotti RS, Kosoy OL, Laven JJ, et al. Genetic and serologic properties of Zika virus associated with an epidemic, Yap State, Micronesia, 2007. Emerg Infect Dis. 2008; 14:1232.

4.Health protection - guidance. Zika Virus. [Internet]. UK; Public Health England. 2015 [last updated 26th April 2017; Cited 2017 June 2]. Available from: https://www . gov. uk/ guidance/zika-virus.

5.European Centre for Disease Prevention and Control. Rapid risk assessment. Zika virus epidemic in the Americas: potential association with microcephaly and Guillain-Barré syndrome. Stockholm, Sweden: European Centre for Disease Prevention and Control; 2015.

6.Hayes EB. Zika virus outside Africa. Emerg Infect Dis. 2009; 15: 1347-50.

7.Musso D, Roche C, Robin E, et al. Potential sexual transmission of Zika virus. Emerg Infect Dis. 2015; 2: 35961.

8.Foy BD, Kobylinski KC, Chilson Foy JL, et al. Probable non-vector-borne transmission of Zika virus, Colorado, USA. Emerg Infect Dis. 2011; 17: 880-82. doi: 10.3201 /eid1705. 101939

9.Dupont Rouzeyrol M, Biron A, O'Connor O, et al. Infectious Zika viral particles in breast milk. Lancet. 2016; 387(10023):1051. doi: 10.1016/S0140-6736(16)00624-3. Epub 2016 Mar 2.

10.Centres for Disease Control and Prevention. Zika Virus. [Internet]. Atlanta; US Department of Health and Human Services. 2016. [Cited 2017 June 12]. Available from: http://www.cdc.gov/zika/geo/index.html

11.Duffy MR, Chen TH, Hancock WT, et al. Zika virus outbreak on Yap Island, Federated States of Micronesia. N Engl J Med. 2009; 360: 2536-43

12.Oehler E, Watrin L, Larre P, et al. Zika virus infection complicated by Guillain Barre syndrome - case report, French Polynesia, December 2013. Euro Surveill. 2014; 19: 4-6. 
13.Pan American Health Organization / World Health Organization. Epidemiological Update: Neurological syndrome, congenital anomalies and Zika virus infection. [Internet]. Washington; PAHO/WHO. 2016. [Cited 2017 June 12]. Available from: http:// www. paho.org/hq/index.php?option=com_docman\&task=doc_vie w\&Itemid=270\&gid=32879 \&lang=en

14.Petersen EE, Staples JE, Meaney-Delman D, et al. Interim Guidelines for Pregnant Women During a Zika Virus Outbreak - United States, 2016. MMWR Morb Mortal Wkly Rep. 2016; 65: 30-33. Doi:

http://dx.doi.org/10.15585/mmwr.mm6502e1

15.Besnard M, Lastere S, Teissier A, Cao-Lormeau V, Musso D. Evidence of perinatal transmission of Zika virus, French Polynesia, December 2013 and February 2014. Euro Surveill. 2014; 19: 13-6.

16.Cauchemez S, Besnard M, Bompard P, et al. Association between Zika virus and microcephaly in French Polynesia, 2013-15: a retrospective study. Lancet. 2016; 387(10033): 2125-32.

17.CDC. CDC health advisory: recognizing, managing, and reporting Zika virus infections in travelers returning from Central America, South America, the Caribbean and Mexico. [Internet]. Atlanta, GA: US Department of Health and Human Services. 2016. [Cited 2017 June 12]. Available from: http://emergency.cdc.gov/han/han00385.asp.

18.Centres for Disease Control and Prevention. Morbidity and Mortality Weekly Report. Possible Association BetweenZika Virus Infection and Microcephaly - Brazil. [Internet]. 2016; 65(3): 59-62. Availabe from: http://www.cdc.gov/ mmwr/volumes/65/wr/ mm 6503e2.htm

19.Sonja A, Rasmussen, Denise J, Jamieson, Margaret A, Honein, et al. Zika Virus and Birth Defects-Reviewing the Evidence for Causality. The New England Journal of medicine. 2016; 374: 1981-87. Doi:

\subsection{6/NEJMsr1604338}

20.WHO. Pregnancy management in the context of Zika virus infection. Interim guidance update. [Internet]. Geneva: WHO. 2016 May 13; WHO/ZIKV/MOC/16.2 Rev.1.

Available from:

http://www.who.int/csr/resources/publications/zika/pregnanc y-management/en/

21.Public Health England. Zika Virus: clinical and travel guidance. [Internet]. 2016. [Cited 2017 June 12]. Available from: http://travelhealthpro.org.uk/insect-tick-biteavoidance/

22.CDC. Travelers' health: avoid bug bites. [Internet]. Atlanta, GA: US Department of Health and Human Services.
2013. [Cited 2017 June 12]. Available from: http://wwwnc. cdc.gov/travel/page/avoid-bug-bites

23.The American Congress of Obstetricians and Gynaecologists. Practice Advisory: Interim guidance of interim care of obstetric patients during Zika virus outbreak. [Internet].Washington, DC: American Congress of Obstetricians and Gynecologist. 2016. . [Cited 2017 June 11]. Available from: http//www.acog.org/ about ACOG/News-room/Practice-advisories

24.Oliveira Melo AS, Malinger G, Ximene R, et al. Zika virus in pregnancy causes brain abnormality and microcephaly. Tip of the iceberg? Ultrasound Obstret and Gynaecol. 2016; 47: 6

25.Hennessey M, Fischer M, Staples JE. Zika Virus Spreads to New Areas - Region of the Americas, May 2015-January 2016. MMWR Morb Mortal Wkly Rep. 2016; 65:55.

26.Petersen EE, Staples JE, Meaney-Delman D, et al. Interim Guidelines for Pregnant Women During a Zika Virus Outbreak - United States, 2016. MMWR weekly report. 2016; 65(2): 30-33.

27.Staples JE, Dziuban EJ, Fischer M, et al. Interim guidelines for the evaluation and testing of infants with possible congenital zika virus infection - United States, 2016. MMWR Morb Mortal Wkly Rep. 2016; 65:63.

28.Victora CG, Schular-Faccini L,Matijasevich A, et al. Microcephaly in Brazil: how to interpret reported numbers? Lancet. 2016 Feb; 387(10019): 621-4.

29. World Health Organization. WHO child growth standards.Methods and development. [Internet]. Geneva: WHO. 2006. [Cited 2017 June 11]. Available from: http://www. who.

int/childgrowth/publications/technical_report_pub/en

30.CDC. Zika virus: Symptoms, Diagnosis, \& Treatment. [Internet]. Atlanta, GA: US Department of Health and Human Services. 2016. [Cited 2017 June 2]. Available from: http://ww.cdc.gov/zika/symptoms/index.html

31.CDC. Interim guidance for the Preconception Counseling and Prevention of Sexual Transmission of Zika virus for persons with possible Zika Virus Exposure - United States. MMWR Morb Mortal Wkly Rep. 2016; 65(39): 1077-81.

\section{Moushmi B. Tadas Parpillewar ${ }^{1}$}

${ }^{1}$ Associate Professor, Department of Obstetrics and Gynaecology, Government Medical College, Nagpur, Maharashtra, India. 